\title{
ON THE WEYL SPECTRUM OF A HILBERT SPACE OPERATOR
}

\author{
JOHN V. BAXLEY
}

\begin{abstract}
Using the perturbation definition of the Weyl spectrum, conditions are given on a closed (possibly unbounded) linear operator $T$ in a Hilbert space which allow the Weyl spectrum to be characterized as a subset of the spectrum of $T$.
\end{abstract}

1. Introduction. Let $T$ be a closed linear operator with domain $D(T)$ dense in a Hilbert space $H$. Let $\sigma(T)$ denote the spectrum of $T, \pi_{0}(T)$ the set of eigenvalues of $T, \pi_{0 \mathrm{f}}(T)$ the set of eigenvalues of finite geometric multiplicity of $T$, and $\pi_{00}(T)$ the set of isolated eigenvalues of finite geometric multiplicity of $T$. (Here, "isolated" means isolated as points in $\sigma(T)$.) Thus

$$
\pi_{00}(T) \subset \pi_{0 \mathrm{f}}(T) \subset \pi_{0}(T) \subset \sigma(T) .
$$

In 1909, H. Weyl [10] investigated the behavior of the spectrum of $T$ under perturbation by compact operators and proved that if $T$ is bounded and selfadjoint, then

$$
\bigcap\{\sigma(T+K): K \text { compact }\}=\sigma(T)-\pi_{00}(T) .
$$

For $T$ an arbitrary closed linear operator, we denote the left-hand side of the above equation by $\omega(T)$ and call $\omega(T)$ the Weyl spectrum of $T$. Recently, several authors ([1]-[5], [7]) have proved that $\omega(T)=\sigma(T)-$ $\pi_{00}(T)$ under conditions on $T$ more general even than normality. All these authors except Bouldin assume that $T$ is bounded with $D(T)=H$. In [3] and [4], Bouldin investigated several alternative definitions of the essential spectrum for a closed linear operator $T$ and in particular gave conditions under which $(*)$ holds. In addition, he considered the effect of replacing the concept of geometric multiplicity by that of algebraic multiplicity.

In this note, we extend the results of [1] on bounded operators to the unbounded case. As in [1], we continue to use the classical definition of the

Presented to the Society, March 31, 1972; received by the editors August 23, 1971 and, in revised form, October 22, 1971.

AMS 1970 subject classifications. Primary 46C10, 47A10; Secondary 47A55.

Key words and phrases. Weyl spectrum, Hilbert space operator, eigenvalue, algebraic multiplicity, geometric multiplicity, perturbation, compact operators. 
Weyl spectrum given above rather than the currently more fashionable definition in terms of Fredholm operators. In $\S 2$, the nature of the Weyl spectrum $\omega(T)$ is investigated for an arbitrary closed linear operator $T$. In $\S 3$, a hypothesis on $T$ is formulated which implies a modified form of $(*): \pi_{00}(T)$ must be replaced by the set of isolated eigenvalues of finite algebraic multiplicity. A similar result in [4] is then obtained as a corollary. Finally, in $\$ 4$, we give conditions under which (*) is valid.

I would like to record here my appreciation to Richard Bouldin for stimulating correspondence and critical remarks.

2. Some general properties of the Weyl spectrum. Throughout this section $T$ is a closed linear operator with domain $D(T)$ dense in a Hilbert space $H$.

We define the algebraic multiplicity of an isolated point $\lambda \in \sigma(T)$ as in [3]. For such a $\lambda$ it is known that there exists a direct sum decomposition $H=A(\lambda) \oplus B(\lambda)$, each summand of which is invariant under $T-\lambda I$; the restriction $N_{\lambda}$ of $T-\lambda I$ to $A(\lambda)$ is bounded and quasi-nilpotent and the restriction $S_{\lambda}$ of $T-\lambda I$ to $B(\lambda)$ has a bounded inverse defined over all of $B(\lambda)$. The dimension $\operatorname{dim} A(\lambda)$ of $A(\lambda)$ is then by definition the algebraic multiplicity of $\lambda$. If $\lambda$ is an eigenvalue of $T$, then the corresponding eigenspace $G(\lambda)$ is contained in $A(\lambda)$ so that the geometric multiplicity $\operatorname{dim} G(\lambda)$ never exceeds the algebraic multiplicity of $\lambda$.

We introduce a little more notation. Let $\alpha(T)$ denote the isolated eigenvalues of $T$ of infinite algebraic multiplicity. Then let $\hat{\pi}_{0 \mathrm{f}}(T)=\pi_{0 \mathrm{f}}(T)-\alpha(T)$ and let $\hat{\pi}_{00}(T)=\pi_{00}(T)-\alpha(T)$. Thus, $\hat{\pi}_{00}(T)$ consists of the isolated eigenvalues of finite algebraic multiplicity.

LEMMA 2.1. $\sigma(T)-\pi_{0}(T) \subset \omega(T)$.

Proof. The simple proof given in [6, Problem 143] for the case that $T$ is bounded generalizes to the present situation.

LEMMA 2.2. $\sigma(T)-\pi_{0 \mathrm{f}}(T) \subset \omega(T)$.

Proof. The proof in [1, Lemma 2] of the same result for $T$ bounded generalizes with no essential change.

LEMMA 2.3. $\sigma(T)-\hat{\pi}_{0 f}(T) \subset \omega(T)$.

Proof. In view of Lemma 2.2, we need only show that $\lambda \in \omega(T)$ if $\lambda$ is an isolated eigenvalue of infinite algebraic multiplicity but finite geometric multiplicity. For such a $\lambda$, it follows from [8, p. 240] that the range of $N_{\lambda}$ is not closed. Hence, the range of $T-\lambda I$ is not closed. If $\lambda \notin \sigma(T+K)$ for some compact $K$, then $(T+K-\lambda I)^{-1}$ is a bounded operator with domain $H$, and so $(T+K-\lambda I)^{-1} K$ is compact. The theory of compact operators 
[9, p. 279] then guarantees that $I-(T+K-\lambda I)^{-1} K$ has closed range. Using the factorization

$$
T-\lambda I=[T+K-\lambda I]\left[I-(T+K-\lambda I)^{-1} K\right],
$$

we easily see that $T-\lambda I$ has closed range, a contradiction.

If $T$ is bounded, we can give a completely elementary proof of this last lemma, without recourse to the theory of compact operators in [9] or [8, p. 240]. Assuming that $A(\lambda)$ is infinite dimensional, let $\left\{e_{n}\right\}$ be an infinite orthonormal sequence in $A(\lambda)$. Suppose $\lambda \notin \sigma(T+K)$ for some compact $K$; then $U=(T+K-\lambda I)^{-1}$ exists as a bounded operator on $H$. Let $E=(2\|U\|)^{-1}$ and choose $k \geqq 2$ so that $\left\|N_{\lambda}^{k}\right\|^{1 / k} \leqq E$. This can be done since $N_{\lambda}$ is quasi-nilpotent. Let $y_{n}=(T+K-\lambda I)^{k} e_{n}$. Then $y_{n}=(T-\lambda I)^{k} e_{n}+V e_{n}$, where $V$ is a sum of $2^{k}-1$ operators each of which is a finite product of bounded operators at least one of which is the compact operator $K$. Thus $V$ is compact. By passing to a subsequence if necessary, we may assume that $y=\lim V e_{n}$ exists. Thus

$$
\left\|y_{n}-y\right\| \leqq\left\|N_{\lambda}^{k} e_{n}\right\|+E^{k} \leqq 2 E^{k}
$$

for $n$ sufficiently large. But $e_{n}=U^{k} y_{n}$ and hence

$$
\left\|e_{n}-U^{k} y\right\| \leqq\|U\|^{k}\left\|y_{n}-y\right\| \leqq 2(E\|U\|)^{k} \leqq \frac{1}{2}
$$

for $n$ sufficiently large. Thus, if $m>n$,

$$
\begin{aligned}
2 & =\left\|e_{n}\right\|^{2}+\left\|e_{m}\right\|^{2}=\left\|e_{n}-e_{m}\right\|^{2} \\
& \leqq\left[\left\|e_{n}-U^{k} y\right\|+\left\|U^{k} y-e_{m}\right\|\right]^{2} \leqq 1,
\end{aligned}
$$

a contradiction.

If $T$ is not bounded, the above proof would be valid if $A(\lambda)$ is invariant under $K$. Otherwise, the operator $V$ might experience severe difficulties.

LEMMA 2.4. $\omega(T) \subset \sigma(T)-\hat{\pi}_{00}(T)$.

Proof. Since $\omega(T) \subset \sigma(T)$, we need only show that $\omega(T) \cap \hat{\pi}_{00}(T)=\varnothing$. Suppose $\lambda \in \hat{\pi}_{00}(T)$. We look for a compact $K$ for which $\lambda \notin \sigma(T+K)$. Since $1 \leqq \operatorname{dim} A(\lambda)<\infty$, the operator $K$ is defined by

$$
\begin{aligned}
K x & =x, & & \text { if } x \in A(\lambda), \\
& =0, & & \text { if } x \in B(\lambda)
\end{aligned}
$$

and extended by linearity to all of $H$ is compact since its range is finite dimensional. Since $N_{\lambda}$ is quasi-nilpotent, then $\sigma\left(N_{\lambda}\right)=\{0\}$ and it follows easily that $\sigma\left(N_{\lambda}+I\right)=\{1\}$. Thus $N_{\lambda}+I$ is one-to-one and onto. Since $S_{\lambda}$ is also one-to-one and onto, it follows that $T+K-\lambda I$ is one-to-one and onto. Thus, $(T+K-\lambda I)^{-1}$ is a closed operator with domain $H$. By the closed graph theorem (see [8] or [9]), $(T+K-\lambda I)^{-1}$ is bounded and $\lambda \notin \sigma(T+K)$. Hence $\lambda \notin \omega(T)$. 
3. Weyl's theorem and algebraic multiplicity. We use the generic phrase "Weyl's theorem" for any theorem which characterizes $\omega(T)$ as a subset of $\sigma(T)$. In this section, we give a sufficient condition that $\omega(T)=$ $\sigma(T)-\hat{\pi}_{00}(T)$.

Condition C-1. If $\left\{\lambda_{n}\right\}$ is an infinite sequence of distinct points in $\hat{\pi}_{0 \mathrm{f}}(T)$, if $\lim \lambda_{n}=\lambda \in \hat{\pi}_{0 \mathrm{f}}(T)$, and if $\left\{x_{n}\right\}$ is a sequence of corresponding normalized eigenvectors, then the sequence $\left\{x_{n}\right\}$ does not converge.

We remark that Condition $\mathrm{C}-1$ is slightly modified from the statement in [1].

THEOREM 3.1. If $T$ satisfies $\mathrm{C}-1$, then $\omega(T)=\sigma(T)-\hat{\pi}_{00}(T)$.

Proof. By Lemma 2.4, we must only show that $\sigma(T)-\hat{\pi}_{00}(T) \subset \omega(T)$. Now $\sigma(T)-\hat{\pi}_{00}(T)=\left[\sigma(T)-\hat{\pi}_{0 \mathrm{f}}(T)\right] \cup\left[\hat{\pi}_{0 \mathrm{f}}(T)-\hat{\pi}_{00}(T)\right]$. By Lemma 2.3, $\sigma(T)-\hat{\pi}_{0 \mathrm{f}}(T) \subset \omega(T)$. Since $\omega(T)$ is closed (topologically),

$$
\operatorname{cl}\left(\sigma(T)-\hat{\pi}_{0 \mathrm{f}}(T)\right) \subset \omega(T) .
$$

Thus, if suffices to show that $\lambda \in \omega(T)$ if $\lambda \in \hat{\pi}_{0 \mathrm{f}}(T)-\hat{\pi}_{00}(T)$ but $\lambda \notin$ $\operatorname{cl}\left(\sigma(T)-\hat{\pi}_{0 \mathrm{f}}(T)\right)$. Thus, there exists an infinite sequence $\left\{\lambda_{n}\right\}$ of distinct points in $\hat{\pi}_{0 \mathrm{f}}(T)$ which converges to $\lambda$. Let $\left\{x_{n}\right\}$ be a sequence of corresponding normalized eigenvectors. Then by Condition C-1, $\left\{x_{n}\right\}$ does not converge. Suppose now that $\lambda \notin \sigma(T+K)$ for some compact $K$. Then $(T+K-\lambda I)^{-1}$ exists as a bounded operator on $H$. Let

$$
y_{n}=(T+K-\lambda I) x_{n}=\left(\lambda_{n}-\lambda\right) x_{n}+K x_{n} .
$$

By passing to a subsequence if necessary, we may assume $y=\lim K x_{n}$ exists. Since $\lim \lambda_{n}=\lambda$, we have $\lim y_{n}=y$. But then

$$
\lim x_{n}=\lim (T+K-\lambda I)^{-1} y_{n}=(T+K-\lambda I) y,
$$

a contradiction.

If $\lambda \in \pi_{0}(T)$, Bouldin [4] says that the eigenspace $G(\lambda)$ corresponding to $\lambda$ is not an asymptotic eigenspace if there exists a $\delta(0<\delta<1)$ such that $|(x, y)| \leqq \delta$ if $x \in G(\lambda),\|x\|=1=\|y\|$, and $y$ is an eigenvector of $T$ corresponding to some eigenvalue $\mu \neq \lambda$. We now get as a corollary to the above theorem a result of Bouldin [4].

COROLlARY. If each finite dimensional eigenspace of $T$ is not an asymptotic eigenspace, then $T$ satisfies Condition $\mathrm{C}-1$ and, consequently, $\omega(T)=$ $\sigma(T)-\hat{\pi}_{00}(T)$.

Proof. Suppose $T$ does not satisfy Condition $\mathrm{C}-1$. Then there exists an infinite sequence $\left\{\lambda_{n}\right\}$ of distinct points in $\hat{\pi}_{0 \mathrm{f}}(T)$ which converges to $\lambda \in \hat{\pi}_{0 \mathrm{f}}(T)$ and a sequence $\left\{x_{n}\right\}$ of corresponding normalized eigenvectors which converges. Let $x=\lim x_{n}$. Then $\lambda x=\lim \lambda_{n} x_{n}=\lim Y x_{n}$. Since $T$ is 
closed, $x \in D(T)$ and $T x=\lambda x$. Thus, $x \in G(\lambda)$ and clearly $\|x\|=1$. Therefore, $\lim \left(x, x_{n}\right)=(x, x)=1$ and $G(\lambda)$ is an asymptotic eigenspace.

4. Weyl's theorem and geometric multiplicity. We now give sufficient conditions in order that $\omega(T)=\sigma(T)-\pi_{00}(T)$. If $T$ satisfies Condition C-1, we already know that $\omega(T)=\sigma(T)-\hat{\pi}_{00}(T)$. Thus, any condition which implies that $\hat{\pi}_{00}(T)=\pi_{00}(T)$ is of interest. We now consider

Condition C-2. If $\lambda \in \pi_{00}(T)$, then $T-\lambda I$ has closed range.

We remark that Condition C-2 above assumes less than the corresponding condition of [1].

THEOREM 4.1. If T satisfies Condition C-2, then $\hat{\pi}_{00}(T)=\pi_{00}(T)$.

Proof. It suffices to show that $\pi_{00}(T) \subset \hat{\pi}_{00}(T)$. If $\lambda \in \pi_{00}(T)$, then $\lambda$ is an isolated eigenvalue of finite geometric multiplicity and thus the null space of $T-\lambda I$ is finite dimensional. By Condition C-2, $T-\lambda I$ also has closed range. Thus, the null space of the quasi-nilpotent operator $N_{\lambda}$ is finite dimensional and $N_{\lambda}$ has closed range. It follows from [8, p. 240] that $\operatorname{dim} A(\lambda)<\infty$.

Corollary. If $T$ satisfies both Conditions $\mathrm{C}-1$ and $\mathrm{C}-2$, then $\omega(T)=$ $\sigma(T)-\pi_{00}(T)$.

This corollary, even if $T$ is bounded, is stronger than the result in [1]. Moreover, the results of Bouldin [3, Theorem 4 and Corollary 3] corresponding to Theorem 4.1 above are proved only in the case that $T$ is bounded. In this case, the referee has pointed out that the condition (5) in [3, Corollary 3] is equivalent to $\operatorname{dim} A(\lambda)<\infty$, which in turn is equivalent (by a theorem of Kato) to our Condition C-2. Thus, [3, Corollary $3]$ in conjunction with [4, Theorem 3] is, at least in the bounded case, essentially equivalent to our corollary above.

That the corollary above contains most known results is adequately documented in [3] and [1]. However, it is known [5] that $\omega(T)=\sigma(T)-$ $\pi_{00}(T)$ holds for any Toeplitz operator. Although C-2 is vacuously satisfied in this case, Richard Bouldin has shown me an example (the adjoint of the unilateral shift on the Hardy space $H_{2}$ ) for which C-1 is violated. Thus, Weyl's theorem for Toeplitz operators lies deeper than any of the general theorems so far discovered.

\section{REFERENCES}

1. J. V. Baxley, Some general conditions implying Weyl's theorem, Rev. Roumaine Math. Pures Appl. 16 (1971), 1163-1166.

2. S. K. Berberian, An extension of Weyl's theorem to a class of not necessarily normal operators, Michigan Math. J. 16 (1969), 273-279, MR 40 \#3335. 
3. R. H. Bouldin, Essential spectrum for a Hilbert space operator, Trans. Amer. Math. Soc. 163 (1972), 437-445.

4. - The Weyl essential spectrum, Proc. Amer. Math. Soc. 28 (1971), 531-536.

5. L. A. Coburn, Weyl's theorem for nonnormal operators, Michigan Math. J. 13 (1966), 285-288. MR 34 \#1846.

6. P. R. Halmos, A Hilbert space problem book, Van Nostrand, Princeton, N.J., 1967. MR 34 \#8178.

7. V. Istrățescu, Weyl's theorem for a class of operators, Rev. Roumaine Math. Pures Appl. 13 (1968), 1103-1105. MR 38 \#6381.

8. T. Kato, Perturbation theory for linear operators, Die Grundlehren der math. Wissenschaften, Band 132, Springer-Verlag, New York, 1966. MR 34 \#3324.

9. A. E. Taylor, Introduction to functional analysis, Wiley, New York, 1958. MR 20 \#5411.

10. H. Weyl, Über beschrankte quadratische Formen deren differenz vollstetig ist, Rend. Circ. Mat. Palermo 27 (1909), 373-392.

Department of Mathematics, Wake Forest University, Winston-Salem, North Carolina 27109

Current address: Department of Mathematics, University of Illinois, Urbana, Illinois 61801 